\title{
L'utilisation des jeux autonomes dans un dispositif hybride pour développer l'apprentissage de la compréhension orale en français langue étrangère
}

\author{
RANA KANDEEL \\ Université du Yarmouk, Jordanie
}

Reçu: 31 août 2015 / Accepté: 12 février 2018

ISSN: $1697-7467$

\begin{abstract}
RÉSUMÉ: Cet article vise à décrire et à analyser l'utilisation des jeux autonomes sur le site TV5monde.com dans un cours de compréhension orale proposé par le département des langues modernes à l'université du Yarmouk en Jordanie. Pour réaliser cet objectif, nous avons soumis un questionnaire aux étudiants ayant expérimenté ces jeux en classe du français langue étrangère (FLE) afin de répondre aux questions concernant l'utilisation des jeux dans l'apprentissage de la compréhension orale. Nous présenterons les apports didactiques de leur utilisation dans un dispositif hybride à cet apprentissage en FLE.

Mots-clés: jeux autonomes, Français langue étrangère (FLE), compréhension orale, dispositif hybride, apprentissage.
\end{abstract}

Using Online Games in a Hybrid Context for the Purpose of Developing the Oral Comprehension Learning in French as a Foreign Language

\begin{abstract}
This paper aims at describing and analyzing using online games on the website TV5monde.com in the oral comprehension lecture at the department of modern languages at Yarmouk University. In order to achieve this goal, we have distributed a questionnaire to students who have tried using these games in classes of French as a foreign language (FFL), where students had to answer questions related to using games in learning oral comprehension. In this article, we will present the didactical contributions of using these games in a hybrid context in this learning in FFL.

Keywords: online games, French as a foreign language (FFL), oral comprehension, hybrid context, learning.
\end{abstract}

\section{INTRODUCTION}

Les jeux en ligne ont des potentialités (multimodalité, auto-correction,etc.) en didactique des langues étrangères et secondes. Nous en évoquons aussi l'exposition à la langue étrangère, l'apprentissage de nouveau vocabulaire dans des situations formelles ou informelles, l'opportunité de développer la compréhension orale, l'intérêt culturel des jeux, l'engagement et le plaisir suscités chez les apprenants (Peterson, 2013: 129, Reinders et Wattana, 2012: 164, Mangenot, 2002: 142). Cependant, ces avantages ne 
peuvent être pensés que dans un cadre d'étude prenant en compte les pratiques des apprenants-utilisateurs de ces outils pédagogiques. Selon une approche sociotechnique, centrée sur le jeu comme objet à la fois technique et social, certains auteurs insistent sur l'importance du retour sur les pratiques et les conditions de la mise en œuvre des jeux en ligne insuffisamment étudiés par les travaux existants (Zabban, 2012: 162). En didactique des langues, y compris celle du FLE, les publications qui sont à l'origine des mises en pratique des jeux ne sont pas complétement empiriques (Silva, 2008 cité par Schmoll, 2016). Or, les études les plus récentes s'intéressent encore à la distinction entre les jeux traditionnels et ceux numériques plutôt qu'aux pratiques des apprenants-joueurs des jeux numériques (Schmoll, 2016, Buzy-Christmann et al., 2016). C'est pourquoi nous nous intéressons dans cet article à l'étude de l'utilisation des jeux en ligne en tenant compte de la relation qui peut être établie entre le jeu, les apprenants-joueurs et l'apprentissage. Autrement dit, la relation entre objets, humains, et sens.

\section{Cadre conceptuel}

Le jeu en classe de langue peut être traditionnel ou numérique. Dans le champ des technologies de l'information et de la communication, les «jeux autonomes» empruntés à De Prato (2012:57) renvoient aux jeux à un joueur individuel, accessibles grâce à un navigateur web, sans avoir à installer de logiciels additionnels. Appelés aussi «jeux de navigateur», ces derniers ont l'avantage de permettre de jouer dans l'espace et dans le temps gratuitement sans aucune contrainte. Ces jeux, exploitables en didactique des langues pour travailler la compréhension orale, sont majoritairement manifestes sous plusieurs formes (activités ludiques ou jeux) sur la Toile. Il est possible d'en distinguer trois catégories sur des sites avec:

- des activités ludiques et des jeux conçus autour des supports authentiques audio -visuels (TV5monde.com).

- Des activités de compréhension orale conçues autour de supports audio fabriqués pour des fins pédagogiques (applications web, bonjour de France).

- des activités ludiques et des jeux complétant les manuels sur les sites compagnons (Bravo, Didier, Hachette).

Nous nous intéressons au premier type de jeux sur le site de TV5monde.com dont le matériel et les structures ludiques fournis sont de qualité et dont les activités sollicitent les compétences langagières de communication (Silva, 2013: 120).

La compréhension est définie comme «l'aptitude résultant de la mise en cuvre de processus cognitifs, qui permet à l'apprenant d'accéder au sens d'un texte qu'il écoute (compréhension orale) ou lit (compréhension écrite)» (Cuq, 2003: 49). Les jeux doivent favoriser la mobilisation des stratégies cognitives dans l'apprentissage de la compréhension 
orale en s'exposant aux documents multimodaux représentant des situations suffisamment diverses. Cette activité langagière suppose «la connaissance du système phonologique, la valeur fonctionnelle et sémantique des structures linguistiques, mais aussi la connaissance des règles socio-culturelles» (Desmons et al., 2005: 27). Les jeux conduisent à un enrichissement phonologique et lexical quand l'apprenant répète les répliques présentées par le système informatique, mémorise un lexique sans lequel il ne pourrait pas avancer pour savoir le sens des mots (Mangenot, 2002: 142). Les fréquents visionnages des documents supports des jeux ou la répétition de ceux-ci assurent une mémorisation implicite du lexique (Courtillon, 2003: 58). Les apprenants peuvent faire des écoutes et des réécoutes individuelles quand ils ne jouent pas d'une façon collective pour exercer l'oreille à la perception sonore ainsi que développer une capacité de discrimination auditive, souvent jugée comme une opération délicate pour des apprenants de langue étrangère. C'est ainsi qu'en français les problèmes de la perception auditive comme «la perception des sons, du débit, des accents, les enchaînements de sons issus de lettres - d'habitude muettes-au sein du groupe rythmique» (Honnay et al., 2013: 69) peuvent être diminués.

L'introduction de ces jeux permet de prendre en compte des dimensions non langagières dans l'apprentissage de langue comme l'attitude ludique de l'apprenant. Cette attitude existe lorsque l'apprenant procède à l'agencement d'un ensemble de règles et de moyens, d'un monde fictionnel et d'un contexte pragmatique (Genvo, 2013). Elle motive les apprenants qui deviennent des joueurs jouants. Silva (2008: 19) fait une distinction fondamentale entre ceux-ci et les joueurs joués. Les premiers participent activement au jeu, et ils le maitrisent contrairement aux joueurs joués qui sont entraînés dans une dynamique qui les dépasse, sans avoir une attitude ludique ou des moyens pour infléchir le jeu.

Dans ce sens, la distraction et le plaisir suscités par le jouer (activité), un procès métaphorique de la décision de mettre en œuvre de schèmes perçus comme aléatoires pour la réalisation d'un thème posé comme arbitraire (Henriot, 1989: 300) créent un contexte favorable au développement d'une attitude ludique. Le jouer véhicule les avantages reconnus au jeu en évitant de le transformer en un simple exercice d'apprentissage. Ce processus permet de sortir le jeu d'un cadre à visée d'apprentissage et de le transformer à un moyen de divertissement comme le souligne Brougère dans son entretien avec Fournier:

Il faut sortir du mythe que le jeu est une situation exceptionnelle: comme dans toutes les activités (y compris le travail), si l'on apprend parfois, l'on n'apprend pas toujours. Parfois, on ne fait que mobiliser des connaissances déjà acquises et le seul but est de réussir. Qu'est-ce que l'on apprend quand on fait une réussite aux cartes par exemple? Oui, le jeu peut n'être qu'un pur divertissement (Brougère et Fournier, 2014).

Enfin, puisque les caractéristiques intrinsèques des jeux autonomes ne manquent pas de potentialités en didactique des langues, nous pensons qu'une vérification de leur efficacité tient principalement à l'usage que l'on en fait. 


\section{Problematique de la recherche}

Le contexte de la formation où se déroule le cours de la compréhension orale est un programme d'étude de français langue étrangère dans le cadre des études de licence dans une université Jordanienne, l'université du Yarmouk. Ce cours s'étend sur quarante-huit heures d'apprentissage dans tout le semestre. Le français est la deuxième langue étrangère apprise après la langue anglaise. Les étudiants inscrits dans ce cours se confrontent à différents problèmes. Nous trouvons, entre autres, l'incapacité de comprendre les documents audio des manuels ${ }^{1}$ parce que ces documents sont considérés comme étant trop rapides dans l'écoute, et la difficulté de la perception auditive des sons. Le manque d'habitude d'écoute chez ce public, la méconnaissance des stratégies d'écoute et l'insuffisance de bagage lexical freinent la compréhension. Ces problèmes sont dus au nombre réduit des cours consacrés à l'apprentissage de la compréhension orale (deux cours en licence) ainsi qu'à la rareté des moments de pratique de cette activité langagière de réception. De plus, les apprenants ne travaillent que sur les thèmes du manuel introduits par les enseignants en utilisant le CD du manuel. Ils réalisent les activités conçues pour des finalités pédagogiques sans recourir aux documents authentiques.

Nous nous sommes partie des problèmes rencontrés par les apprenants en mettant en place un dispositif intégrant les jeux dans l'apprentissage afin d'améliorer l'apprentissage de la compréhension orale, une orientation méthodologique préconisée par certains spécialistes des langues (Puren, et al., 2013: 127). En effet, ces auteurs évoquent que les technologies doivent apporter des solutions aux problèmes rencontrés par les enseignants.

Notre problématique porte donc sur l'utilisation des jeux autonomes dans le développement de l'apprentissage de la compréhension orale. Nous nous intéressons tout particulièrement à savoir les apports didactiques de leur utilisation et de savoir ce que les apprenants pensent de ces jeux. L'exploitation de ce type de jeux acquiert une certaine légitimité pour diversifier les méthodologies d'enseignement et les ressources pédagogiques permettant de placer l'apprenant au centre de l'apprentissage et d'utiliser des processus d'enseignement plus adaptées à leurs besoins et plus motivants. Ces jeux ont été contextualisés selon trois niveaux: un contexte général de la formation (cours de compréhension orale), un contexte technopédagogique spécifique au cours (dispositif hybride) et un contexte plus étroit aux jeux (ateliers de langue française sur le site de TV5 monde.com).

\section{LES PROFILS DES ETUdIANTS}

Les étudiants participant à ce cours sont au nombre de seize. Ce public est homogène quant au niveau de leur apprentissage du FLE (débutants du niveau A2 selon les niveaux du CECRL) et au niveau de leurs âges (19-20 ans). Ils ont tous l'arabe comme langue maternelle et ils ne possèdent aucune connaissance de la langue française avant son apprentissage à l'université. Ils sont de nationalité jordanienne et ils sont en deuxième année de licence en langues vivantes.

\footnotetext{
${ }^{1}$ M. Barféty, P. Beaujouin (2005). Compréhension orale, niveau 2, Clé international, Paris.
} 


\section{SCENARISATION DU DISPOSITIF D'APPRENTISSAGE}

La scénarisation du dispositif se fait en tenant compte de la réorganisation de l'espace et du temps de l'apprentissage, du choix des jeux, des modalités d'accompagnement humain et de l'évaluation.

\subsection{Le dispositif hybride}

Ce dispositif semble le plus pertinent dans la mesure où il offre un environnement technopédagogique (Charlier et al., 2006: 470) capable d'améliorer les conditions et la qualité de la formation à la compréhension orale. Son caractère hybride articulant à la fois des séances de travail en présentiel et à distance, et combinant un apprentissage formel et informel, individuel et collectif permet d'introduire de nouvelles démarches pédagogiques.

En présentiel, les étudiants travaillent sur les leçons du manuel. Quant au mode distanciel, ils sont invités à effectuer les trois séries de jeux que nous avons proposés pendant tout le semestre, soit quatre mois. Chaque jeu est lié à un thème du manuel abordé pendant le cours, et après la présentation de ce thème en présentiel, les étudiants ont deux semaines pour faire ces jeux en distanciel. En allant sur la page de notre formation sur le système du e-learning installé par l'université qui utilise la plateforme Moodle, les étudiants inscrits dans le cours ont tous accès au dispositif.

\subsection{Les jeux de l'atelier «une journée chez Seekube»}

Les jeux mis en œuvre dans notre dispositif de form ation sont disponibles dans les ateliers du sous-rubrique «Parlons français, c'est facile!» dans la rubrique de la langue française du site TV5monde.com ${ }^{2}$. Les ateliers sont regroupés, en général, selon les niveaux des apprenants (débutant, élémentaire, moyen, avancé) et les jeux utilisés dans le cours sont destinés à un public de niveau élémentaire. Nous avons proposé trois séances d'activités ludiques à distance dans le cadre de dispositif hybride présenté dans les lignes qui suivent. L'espace limité dont nous disposons nous amène à décrire seulement une série de jeux de l'atelier «une journée chez Seekube», composée de trois jeux:

- «Bavardons-Culture-Lexique-Compréhension»: ce jeu de simulation et interactif demande au joueur de prendre part à une conversation avec un personnage qui travaille dans une entreprise. Le joueur doit écouter ou lire la consigne qui va permettre de poser une question ou y répondre et il peut aussi écouter la réponse qui apparaît sous forme d'énoncés à choix multiples.

- «Attrape-mot-Culture-Grammaire-Lexique»: la haute interactivité de ce jeu conduit le joueur à connaître les mots à attraper après la lecture des consignes en déplaçant un aspirateur pour attraper ces mots. En cas de réponse incorrecte, le déplacement de l'aspirateur devient moins rapide.

\footnotetext{
${ }^{2}$ Les activités de compréhension orale dans les ateliers sur le site TV5monde http://www.tv5monde.com/
} 
- «Tir au mot-Culture-Lexique»: le joueur doit garder des mots et détruire d'autres, les cibles, par un outil de tirage en fonction de la consigne fournie. Pour répondre à la consigne «garder les verbes sans pronom», il doit tirer sur tous les verbes pronominaux pour éliminer les mots intrus. C'est un jeu pour travailler la grammaire et le lexique verbal.

Pour réaliser les jeux, il faut d'abord visionner et comprendre le webdoc autour duquel ils sont organisés, puis faire les exercices de la compréhension orale avant de faire le jeu. Un dispositif médiatisé d'accompagnement en ligne est mis en place par le site TV5 monde.com.

\subsection{Approche pédagogique et accompagnement humain}

L'objectif de ce dispositif n'étant pas de transformer tout le travail sur la compréhension orale en jeu parce qu'il y a des moments où on ne joue plus dans l'apprentissage (Meirieu, 2006: 3). L'accompagnement humain en présentiel est de type cognitif, linguistique, pédagogique et affectif. Il permet de transférer des stratégies d'écoute et d'identifier les éléments paralinguistiques facilitant la compréhension. L'acquisition de nouveau vocabulaire et le travail sur l'expression orale se font aussi dans les séances présentielles, car le département exige de l'enseignant de proposer des activités d'expression orale dans le cours de la compréhension orale. En effet, le présentiel permet mieux, au niveau affectif, d'encourager la spontanéité de l'expression et de combler les lacunes suscitées par le manque d'interaction à distance quand ils jouent de façon individuelle.

A distance, nous avons réparti les jeux dans les semaines de la formation sur la plateforme après y avoir joués nous-même plusieurs fois pour les prendre en main et vérifier leur adéquation à nos objectifs d'enseignement ainsi qu'aux niveaux linguistiques et besoins des apprenants. Nous avons proposé les consignes de chaque série où l'apprenant trouve le numéro de l'atelier de jeu, le niveau visé, le lien vers les jeux ${ }^{3}$, la durée de leur réalisation. L'envoi à l'enseignante des solutions des jeux proposées par chaque apprenant via la plateforme Moodle était demandé pour vérifier la participation des apprenants et pour créer une ambiance de compétition entre les participants. L'insistance sur l'aspect ludoaffectif n'était pas absent encore là, l'énoncé «Amusez-bien en jouant!» a été inclus dans la consigne.

\subsection{Evaluation}

L'évaluation dans le dispositif d'apprentissage a été double: une évaluation automatisée fournie par le système informatique de type formatif et sommatif et une rétroaction sur l'activité réalisée. Une fois les solutions des jeux sont achevées en ligne, une deuxième évaluation est faite de ces mêmes solutions envoyées à l'enseignant sous forme de document Word. L'enseignant accorde une note à leur réalisation afin de valoriser les activités des étudiants et de créer une ambiance concurrente pleine de défis, ce qui comble les lacunes du mode de travail solitaire.

3 http://parlons-francais.tv5monde.com/webdocumentaires-pour-apprendre-le-francais/Ateliers-de-francais/p-93-1g0-Ateliers-elementaires.htm?pageliste $=2$ 


\section{Methodologie de la recherche}

Nous avons distribué un questionnaire (voir annexe 1) de type question/réponse ouverte après la réalisation de la deuxième série de jeux, c'est-à-dire deux mois et demi après le début du premier semestre en 2014. Les questions sont posées pour connaître des éléments sur l'utilisation des jeux dans l'apprentissage de la compréhension orale tels que l'appréciation des jeux par les étudiants, leurs préférences; les jeux les plus et les moins aimés, les raisons d'adhésion ou de rejet de ces outils, les difficultés rencontrées dans la réalisation des jeux et l'apport du dispositif hybride à l'amélioration de l'apprentissage de la compréhension orale. Nous avons distribué le questionnaire aux seize étudiants inscrits dans le cours, mais nous avons eu le retour de dix étudiants.

L'analyse des réponses est qualitative, il s'agit d'une analyse de contenu des discours des étudiants selon des catégories d'analyse thématique. Les réponses ont étaient recueillies en arabe, puis traduites en français. Nous avons donné un nombre à chaque étudiant afin de garder l'anonymat des sujets.

\section{Categories d’analyses}

Nous avons regroupé les principaux résultats selon quatre catégories d'analyses: l'appréciation des jeux, les difficultés de leur réalisation, la manière de développement de l'apprentissage de la compréhension orale, et la perception des étudiants des pratiques de jouer et du dispositif.

\subsection{L'appréciation des jeux}

\subsubsection{Le(s) jeu(x)le(s) plus aimé(s) par les étudiants}

Les étudiants ont exprimé leur préférence pour le deuxième jeu appelé par eux «le jeu de feu». L'acquisition de nouveau lexique et le sentiment de s'amuser étaient prédominants dans leur discours. Par exemple, E6 dit que: " c'était utile pour savoir le nouveau vocabulaire», et E8 évoque que: «j'ai aimé le deuxième jeu car j'ai acquis plusieurs nouveaux mots surtout dans les verbes pronominaux».

La répétition des expressions telles que «il était amusant» par E3, E7, «je me suis bien amusée en jouant» E1, «je me suis divertie» E10, était un indice clair sur l'attitude ludique apparue dans leurs actions. Ce qui crée le divertissement c'est la combinaison des différents aspects tels que l'attractivité du matériel ludique du jeu comme le dit E2 «j'ai aimé le deuxième jeu car l'aspirateur qui attrape les mots corrects m'a plu», et le défi de sa réalisation suscité par le système de notification dans le jeu car le joueur est obligé de jouer pour obtenir un meilleur score, selon E3: «j'ai aimé dans ce jeu le défi et l'obligation de jouer jusqu'à l'obtention de la meilleure note». 


\subsubsection{Le(s) jeu(x)le(s) moins aimé(s) par les étudiants}

Les discours des étudiants sur le jeu le moins aimé ont varié. Certaines réponses affirmant que tous les jeux étaient amusants et interactifs et qu'ils leur ont permis d'avoir de nouvelles informations sur la langue (E 1, E6, E8, E9), d'après E6: «Aucun jeu. J'ai aimé tous les jeux parce qu'ils m'ont apporté de belles informations». Ces réponses viennent renforcer le discours précédent de la majorité des étudiants sur les raisons d'aimer les jeux.

Par contre, d'autres propos évoquent que le premier jeu était difficile (E2, E3, E10) et c'est cette difficulté qui ne leur a pas conduit à aimer le jeu. Les difficultés dans ce jeu sont éprouvées à deux niveaux, linguistique et ludique. En premier lieu, il était difficile de comprendre le discours de la vidéo, le support à partir duquel les étudiants ont dû répondre aux questions. En deuxième lieu, les questions et les réponses proposées par le jeu ont un niveau plus élevé que celui de certains étudiants, selon E2: «Je n'ai pas aimé les questions, j'ai senti qu'elles ont un niveau plus élevé que le mien».

La difficulté ludique est apparue au niveau de la contrainte du jeu qui consiste à être recommencé par l'étudiant quand il dépasse le nombre d'essai permis dans la réalisation de jeu sans erreurs, selon E3: «Le jeu qui est joué sous forme de questions/réponses parce que quand vous avez deux fois de réponses incorrectes, il a fallu recommencer le jeu».

\subsection{Les difficultés rencontrées dans la réalisation des jeux}

La majorité des étudiants a parlé des difficultés (E2, E3, E5, E6, E8, E9, E10) de compréhension des règles de jeu et du processus à suivre pour la faire. E5, E2 soulignent qu': «il était difficile de comprendre ce qu'il faut faire pour jouer le jeu». Un seul étudiant a expliqué que le vocabulaire était difficile pour lui, selon E 9: «Dans le deuxième jeu, je l'ai répété plusieurs fois car le vocabulaire était difficile à comprendre son sens».

La longue durée de la réalisation de jeu est évoqué aussi parmi les difficultés rencontrées par E8, E3: «le jeu de feu qui était difficile et il a pris beaucoup de temps pour le jouer». L'obligation de la répétition des jeux quand l'étudiant fait des erreurs a été perçue comme une difficulté pour eux comme l'explique E8: «la seule difficulté était celle du temps et le système des jeux, c'est-à-dire vous perdez si vous avez deux erreurs et vous serez obligé de refaire le jeu». Les problèmes techniques sont aussi présents dans les discours des étudiants, E6 et E10, évoquent que la connexion d'Internet était lente.

\subsection{La manière du développement de l'apprentissage de la compréhension orale dans le dispositif hybride}

La majorité des étudiants ont dit que l'écoute de la vidéo aide à savoir faire les jeux et à comprendre son thème et le sujet dont il traite comme l'indiquent E3, E1 par «la compréhension du sujet de l'écoute».

La concentration sur l'écoute et la répétition du jeu ont été perçues comme éléments participant au développement de l'apprentissage de la compréhension orale par E1, E3 et E5. À travers l'utilisation de l'icône de l'écoute des questions et des réponses proposées dans le premier jeu, certains étudiants (E6, E9) ont dit avoir la chance de s'entraîner à la prononciation. Selon E8: «j'ai utilisé l'icône de son qui permet d'écouter des mots et des phrases et par la répétition de l'écoute, j'ai senti que j'avais développé la compréhension 
orale». L'écoute de la vidéo a aidé à savoir la façon de parler sur le travail, E3 déclare que "L'écoute de la vidéo était une bonne façon pour savoir les bonnes réponses et la façon de parler sur le domaine de travail».

Les jeux ont participé à développer des stratégies de compréhension par la recherche sur Internet du sens des mots E2 «j'ai écrit les mots que je n'ai pas sus ou compris sur le moteur de recherche Google et j'ai recherché leurs sens dans le dictionnaire en ligne» et par un travail individuel de compréhension des mots sans l'aide de l'enseignant, comme le dit E10: «J'ai essayé de comprendre les mots tout seul». L'alternance des jeux en ligne avec les cours en présentiel a aidé l'étudiant E4 à s'approprier l'activité de l'écoute: «je me suis approprié la compétence de l'écoute en pratiquant les jeux et dans le cours en présentiel».

L'acquisition de nouveau lexique, la compréhension du sens des mots et des phrases et le travail sur la prononciation paraissent les objectifs prioritairement réalisés par les étudiants. Outre ces composantes linguistiques, d'autres composantes de la compétence à communiquer langagièrement évoquées dans le cadre européen commun de référence (CECRL) ont été essentielles pour certains étudiants, comme par exemple les composantes sociologique et pragmatique. La réflexion et le travail sur des éléments pragmatiques (rituel de discours) ont été présents dans le discours d'un étudiant.

\subsection{La perception des étudiants des pratiques de jouer et du dispositif}

Dans les pratiques de jouer, les étudiants ont fait des expériences avec la langue plutôt que de l'étudier (Right, Betteridge \& Buckby, 2006: 2), et certaines caractéristiques ont marqué leur perceptions de jouer et du dispositif, nous en évoquons:

\subsubsection{La facilité et la rapidité de la réalisation des jeux}

Les étudiants ont des représentations sur l'utilisation des jeux dans l'enseignement des langues parmi lesquelles c'est que le jeu doit être facile et rapide à réaliser. Sept étudiants pensent que les jeux doivent se faire sans efforts, selon eux, si le jeu demande la compréhension du support, il devient difficile de jouer. En effet, les étudiants ne sont pas conscients de la différence entre les jeux en ligne et hors ligne, ce qui pourrait démotiver certains d'entre eux quand la mise en pratique des jeux nécessite la compréhension des instructions en langue étrangère. Pour faire face à ce comportement, il est important de les encourager à solliciter une aide ou un conseil de l'enseignant ou de leurs collègues. D'ailleurs, il n'est pas facile pour eux de s'intégrer vite dans un processus réflexif, centré sur la mise en œuvre du traitement cognitif de l'information, compte tenu de leurs habitudes d'apprentissage dans un contexte où l'étudiant reste le récepteur des connaissances, il a moins de chance pour prendre des initiatives.

\subsubsection{La répétition des activités des jeux}

La répétition est un exercice qui a deux acceptations; quand elle est imposée par le jeu à cause des erreurs commises, il est considéré comme un élément négatif. Par contre, quand les étudiants le font pour des raisons de compréhension de la vidéo ou de la maîtrise de la prononciation, il devient alors plus acceptable. Nous constatons que dans les deux cas la 
répétition a pour objectif essentiel de faciliter l'accès au sens de document oral. L'identification des informations et la réécoute tant des messages de la vidéo que des questions-réponses du premier jeu, témoignent des stratégies adoptées pour faciliter la perception auditive, souvent considérée comme une activité difficile surtout pour des apprenants débutants.

La répétition des activités de support et des jeux nous éclaire sur le processus mis en œuvre. En effet, les étudiants ont majoritairement suivi la manière du processus sémasiologique ou bas-haut qui «consiste à discriminer des formes, les segmenter, et à interpréter le sens à partir de ces segments» (Cuq, 2003: 50). Ils dégagent le sens du webdoc pour réaliser les jeux, surtout les jeux du lexique et de la grammaire. Dans le cas du premier jeu, nous avons remarqué que les étudiants ont adopté un processus onomasiologique ou haut-bas quand ils ont utilisé différentes références linguistiques ou socioculturelles pour faire des prévisions hypothétiques de contenus.

\subsubsection{L'organisation du dispositif hybride}

Prévoir la mise en œuvre du jeu individuel en ligne dans le cadre d'un dispositif pédagogique semi-présentiel ou entièrement à distance reste une pratique peu fréquente dans les contextes d'enseignement/apprentissage des langues comme le souligne Silva (2013: 121). Pourtant, cette étude a montré que le dispositif mis en œuvre a eu un impact sur les conditions spatio-temporelles de la réalisation des jeux, ce qui a influé sur l'apprentissage de la compréhension orale. Jouer en ligne n'a pas exigé une réservation de la salle informatique de l'université parce que les étudiants ont joué chez eux et ils ont tous une connexion Internet. De plus, le mode distanciel a augmenté les heures de contact avec la langue française et d'entraînement à la compréhension orale. Les étudiants ont compté au moins deux heures de la réalisation des jeux sans compter le temps consacré à la répétition de l'écoute de la vidéo ou à la recherche sur Internet du sens des mots.

\section{Discussion}

Nous synthétisons notre discussion autour de deux points essentiels: les apports didactiques des jeux à l'apprentissage de la compréhension orale, et l'autonomie et sa relation avec la médiation de l'enseignant.

\subsection{Les apports didactiques des jeux en ligne à l'apprentissage de la compréhension orale}

Les apports didactiques peuvent être classifiés à plusieurs niveaux dans l'apprentissage de langue:

\subsubsection{L'apprentissage de la compréhension orale}

Outre l'acquisition de nouveaux vocabulaire, l'entraînement à la prononciation, les jeux ont permis aux étudiants de s'exposer à une situation de communication où ils en dégagent les comportements linguistiques préparatoires à la prise de parole et ils apprennent un discours en situation mais pas seulement à partir de listes de mots (Desmons et al., 
2005: 26). Or, la pratique des jeux en ligne favorise le développement d'autres stratégies facilitant la compréhension orale des documents comme par exemple, la recherche du sens du mot par l'utilisation des dictionnaires en ligne, l'acquisition des formules du discours par la répétition de la vidéo pour mieux comprendre le sens et les fonctions du langage car la compréhension «n'est pas une simple activité de réception d'un message qu'il faudrait décoder mais la reconnaissance de la signification d'un discours et l'identification de fonctions communicatives» (Ibid, p. 27).

Les jeux ont un double avantage. Premièrement, ils sont adaptables, c'est-à-dire, qu'ils peuvent concerner la compréhension orale globale aussi bien que détaillée. Deuxièmement, ils sont mesurables puisqu'il n'y pas d'ambigüité dans la réponse.

\subsubsection{L'attitude ludique}

L'apparition d'une attitude ludique définie comme la conviction intime du joueur par rapport au sens de ses actes (Silva, 2008: 18) a été visible chez les étudiants qui ont eu la conscience de jouer. L'activité ludique a été pratiquée pour le plaisir qu'elle procure, c'est-à dire qu'ils ont pensé les jeux en attachant le sens de ces derniers à leurs actes d'apprentissage de la compréhension orale. De plus, certains étudiants étaient des joueurs jouants, ils étaient responsables de l'accès aux sens en mobilisant leurs compétences générales et langagières. Bien que l'utilisation de ce type de jeux ait exigé beaucoup d'efforts de certains étudiants, selon leurs niveaux, pour les jouer, leur pratique a préservé les ressorts ludiques spécifiques à ces jeux. Cette conclusion nous conduit à dire que la parution ou non d'une attitude ludique dépend en grande partie du joueur et de son intention de jouer et de la place qu'il réserve à l'activité ludique dans l'apprentissage. Son attitude ludique peut être soutenue par une médiation de l'enseignant organisant et contextualisant son activité de jouer.

\subsection{L’autonomie et la médiation de l'enseignant}

L'utilisation des jeux a conduit à des conclusions concernant l'autonomie. En premier lieu, la nécessité de prendre en compte la relation entre l'apprenant, l'enseignant et l'apprentissage des langues étrangères dans un contexte donné car cette relation influe sur l'autonomie. L'apprenant, en se retrouvant dans un contexte transmettant les savoirs et qui ne le place pas au centre de l'apprentissage, est fortement guidé par l'enseignant. Il a moins la capacité d'avoir une attitude autonome dans la réalisation des jeux. En deuxième lieu, C'est essentiellement la motivation de l'apprenant et sa responsabilité dans l'apprentissage qui contribuent à l'autonomisation dans la pratique des jeux.

Ces deux éléments confirment que l'autonomie dans la pratique des jeux à distance n'est pas perçue de la même façon par tous les étudiants. La majorité des étudiants ont dit avoir faire des efforts pour pouvoir réaliser les jeux, mais d'autres ont montré une attitude dépendante, moins autonome quand ils ont dit qu'ils n'étaient pas capables de réaliser les jeux tous seuls. C'est à partir de ce point qu'une réflexion sur la médiation de l'enseignant et sa relation avec la médiatisation technologique des jeux dans ce dispositif doit être menée et il reste des questions auxquelles il faut répondre: faut-il reformuler les instructions des jeux et leurs règles dans la consigne de l'enseignant dans le dispositif distanciel pourqu'elles soient plus claires pour les étudiants? Ou faut-il laisser les étudiants travailler d'une façon 
autonome pour comprendre les détails des règles des jeux et leurs instructions?

A notre avis, pour réaliser l'objectif d'être indépendant dans l'apprentissage de la langue et d'avoir plus d'heures de contact avec la langue, la deuxième réponse nous semble plus raisonnable pour un public adulte. Une réponse par l'affirmation à la première question risque de conduire les apprenants à travailler dans un environnement très guidée, contredisant les principes de l'apprentissage autonome. Nous pensons que les difficultés rencontrées dans l'apprentissage de langue sont reliées à l'attitude dépendante des étudiants vis-à-vis de l'enseignant. Elles peuvent se réduire en habituant les étudiants à travailler en autonomie. De plus, la médiation que peut faire l'enseignant sur les activités médiatisées des jeux (instructions, explication des consignes, etc.) risque de transformer l'activité ludique en une activité dénouée d'attractivité et des formes ludiques si d'autres détails y sont ajoutés.

\section{CONCLUSION}

$\mathrm{Au}$ terme de cette étude, nous pouvons dire que les apports didactiques de l'utilisation des jeux autonomes à l'apprentissage de la compréhension orale sont reconnus aux niveaux linguistique et ludique. Mais, une intégration adéquate de ces outils nécessite de réfléchir sur les spécificités des contextes d'utilisation et des profils des apprenants et de leurs besoins. En effet, le choix de dispositif hybride a eu un impact sur la variété des processus mobilisés, la diversité des activités, des pratiques, des savoirs et des compétences mis en œuvre par les étudiants-joueurs pour développer cet apprentissage. Ce choix a montré l'importance de se confronter aux situations de communication authentiques et plus contextualisées. Mais, si le jeu peut modifier la vision de la matière enseignée comme le soulignent Cuq et Gruca (2005: 457), le défi de leur intégration dans une classe de langue est majeur. L'enseignant doit savoir ouvrer une médiation pédagogique au sein des environnements ludiques en ligne en développant l'autonomie des apprenants. Les frontières de la médiation doivent être établies aussi de façon à maintenir une attitude ludique chez les apprenants sans l'effacer par le jouer distanciel. Ces conclusions doivent conduire à une réflexion sur la formation des enseignants de langues à la pédagogie des jeux en ligne.

\section{REFERENCES BIBLIOGRAPHIQUES}

Brougère, G. et Fournier, M. (2014). «A quoi sert le jeu? «entretien avec Gilles Brougère, in Magazine sciences humaines, 3, 257, Disponible sur http://www.scienceshumaines.com/, accès 20 juillet, 2015.

Buzy-Christmann, D., et al. (2016). «Correspondances et contrastes entre jeux traditionnels et jeux numériques», in Sciences du jeu, 5. Disponible sur https://sdj.revues.org/547_

Charlier, B. et al. (2006). «Apprendre en présence et à distance. Une définition des dispositifs hybrides», in Distances et savoirs, 4: 469-496.

Courtillon, J. (2003). Élaborer un cours de FLE. Paris: Hachette.

Cuq, J. P. (dir.) (2003). Dictionnaire de didactique du français langue étrangère et seconde. Paris: CLE international. ASDIFLE.

Cuq, J. P. et Gruca, I. (2005). Cours de didactique du français langue étrangère et seconde. Grenoble: Presses Universitaires de Grenoble. 
De Prato, G. (2012). «Les jeux en ligne: un laboratoire de modèles d'affaires», in Réseaux, 3, 173: 53-75.

Desmons, F. et al. (2005). Enseigner le FLE (français langue étrangère) pratiques de classe. Paris: Belin.

Genvo, S. (2013). «Penser les phénomènes de ludicisation à partir de Jacques Henriot», in Sciences du jeu, 1, Disponible sur http://sdj.revues.org/251

Honnay, F.et al. (2013). «L'utilisation d'un outil nomade (iPod?) et TV5MONDE en classe de FLES pour la compréhension orale: quelles adaptations aux stratégies d'écoute?», in Dialogues et cultures, Revue de la Fédération internationale des professeurs de français, 59: 66-74.

Henriot, J. (1989). Sous couleur de jouer. La métaphore ludique, Paris: José Corti.

Mangenot, F. (2002). «L'apprentissage des langues», in D. Legros et J. Crinon (eds.), Psychologie des apprentissages et multimédia. Paris: Armand Colin, 128-153.

Meirieu, P. (2006). «Le désir et la règle». Entretien avec Yvana Ayme, in Cahiers pédagogiques, 448. Disponible sur http://www.meirieu.com/ARTICLES/desiretregle.pdf, accès 10 août, 2015

Peterson, M. (2013). Computer games and language learning. New York: Palgrave Macmillan.

Puren, C., et al. (2013). "Manuels d'apprentissage entre papier et numérique», in Le français dans le monde, Recherches et applications, 54: 122-130.

Reinders, H. et Wattana, S. (2012). Talk to me! Games and students' willingness to communicate, in H. Reinders (ed.), Digital games in learning language and teaching. Basingstoke, UK: Palgrave Macmillan, 156-188.

Right, A. et al. (2006). Games for language learning. Cambridge: Cambridge University Press.

Schmoll, L. (2016). «L'emploi des jeux dans l'enseignement des langues étrangères: Du traditionnel au numérique», in Sciences $d u$ jeu, 5. Disponible sur https://sdj.revues.org/628

Silva, H. (2008). Le jeu en classe de langue. Paris: CLE international.

Silva, H. (2013). «Jouer et apprendre le français langue étrangère grâce à TV5 MONDE», in Dialogues et cultures, Revue de la Fédération internationale des professeurs de français, 59: 118-129.

Zabban, V. (2012). «Retour sur les game studies. Comprendre et dépasser les approches formelles et culturelles du jeu vidéo», in Réseaux, 173-174 (3-4): 137-176. 


\title{
11. AnNeXe: Questionnaire SUR L'Utilisation DeS JeUX
}

\author{
Université du Yarmouk \\ Département des langues modernes \\ Questionnaire sur les jeux en ligne \\ L'atelier « * une journée chez Seekube *
}

Année 2014-2015

L'objectif de ce questionnaire est de recueillir des informations sur votre utilisation des jeux en ligne dans le cours de la compréhension orale afin d'améliorer cette utilisation. Veuillez répondre à toutes les questions suivantes. Votre réponse sera utilisée pour des fins scientifiques, elle sera traitée de façon anonyme.

1. Quel est votre âge ?

2. Vous êtes en quelle année d'étude à l'université ?

3. Combien de temps vous avez jouez avec ces jeux?

4. Quel jeu vous avez aimé le plus ? Pourquoi ?

5. Quel jeu vous n'avez pas aimé ? Pourquoi ?

6. Est-ce que vous avez rencontré des difficultés dans la réalisation des jeux ? lesquelles ?

7. Pensez-vous que le travail en présentiel et à distance vous aide à améliorer votre apprentissage de compréhension orale ? si oui comment ?

- Si non pourquoi ? 